\title{
Oxidation of AllnAs for Current Blocking in a Photonic Crystal Laser
}

${ }^{*}$ R. Zhang ${ }^{1}$, J.J.G.M. van der Tol $^{1}$, H. Ambrosius ${ }^{1}$, P. Thijs ${ }^{3}$, B. Smalbrugge, T. de Vries ${ }^{1}$, G. Roelkens ${ }^{2}$, F. Bordas ${ }^{1}$, and M.K. Smit ${ }^{1}$

${ }^{1}$ COBRA Research Institute, Technische Universiteit Eindhoven Address: P.O. Box 513,5600 MB Eindhoven, The Netherlands

${ }^{2}$ Photonics Research Group, Ghent University-IMEC, Ghent, Belgium

${ }^{3}$ PHILIPS, The Netherlands

*R.Zhang: Email r.zhang@tue.nl

Abstract: To make an electrically pumped photonic crystal membrane laser is a challenging task. One of the problems is how to avoid short circuiting between the $p$ - and n-doped parts of the laser diode, when the membrane thickness is limited to $200-300 \mathrm{~nm}$. We propose to use the oxide of AllnAs to realize a current blocking function. In this way, based on submicron selective area re-growth, we aim for electrically injected photonic crystal lasers with high output power, small threshold currents and low power consumption. Here results are presented on the oxidation of AllnAs. The results show that it is feasible to use the oxide of AllnAs for current blocking in an InPbased membrane photonic crystal laser.

1 Introduction: Wet oxidation of Al containing materials has brought improvements in Vertical Cavity Surface Emitting

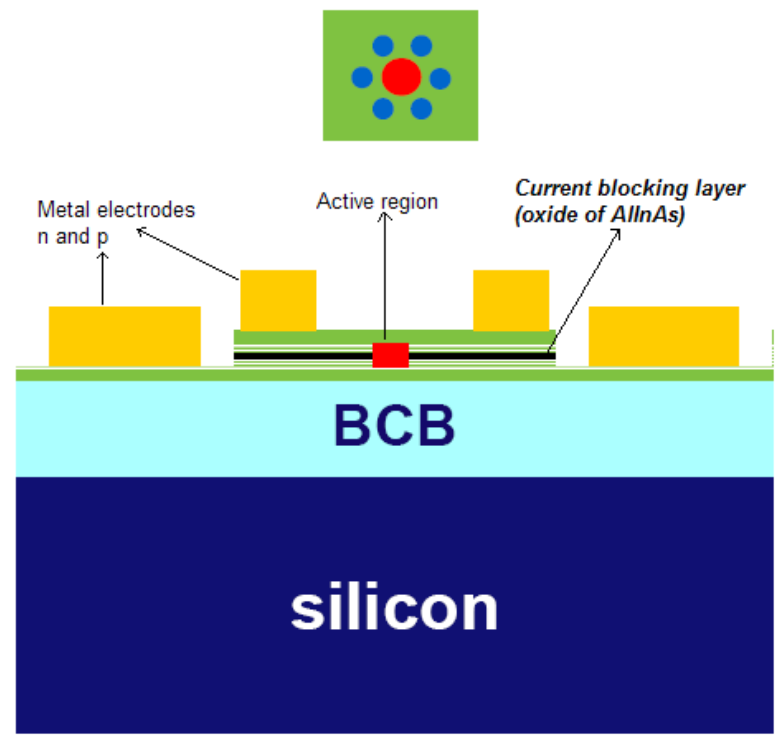

Fig. 1. Schematic view of the proposed electrically injected Photonic Crystal laser

Laser (VCSEL) performance [1]. Uses of this native oxide as a dielectric aperture for optical and current confinement have enabled ultra-low threshold currents and high-output power VCSELs [2]. Building on a submicron selective area re-growth technique [3], we want to use the oxide of AllnAs for current blocking in an $\ln P$ membrane photonic crystal laser (Fig. 1). Due to the high resistance in the oxide, carriers are forced through the active region in the cavity. Thus the pumping efficiency is increased. Meanwhile the threshold current and power consumption will be reduced.

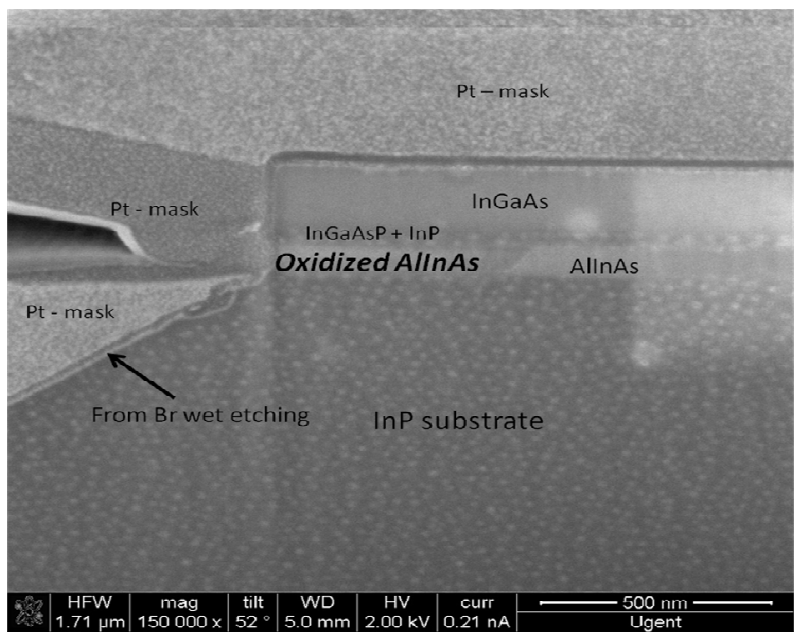

Fig. 2. SEM picture of AllnAs oxide from Focused lon Beam etching

\section{Design and Fabrication}

2.1 Experimental set-up: The oxidation experiment was carried out in a horizontal quartz tube in a furnace. This furnace is fed by nitrogen gas passed through a water bubbler maintained at $95^{\circ} \mathrm{C}$.

2.2 Process optimization: Various kinds of oxidation tests have been done to find the right process window. After several rounds of optimization, good quality of oxidization of the AllnAs is obtained as shown in Fig.2. The oxidation is done at $450^{\circ} \mathrm{C}$ for about $2 \mathrm{hrs}$. The oxidation depth is around $600 \mathrm{~nm}$ which is enough for application in photonic crystal lasers. 


\section{Electrical measurement:}

3.1 The idea: One of the key features of the InP-based membrane platform is its ultra-small thickness (200-300nm)[4]. Therefore the thickness of the oxide layer should be limited to $100 \mathrm{~nm}$. Whether this small thickness of oxide can give high

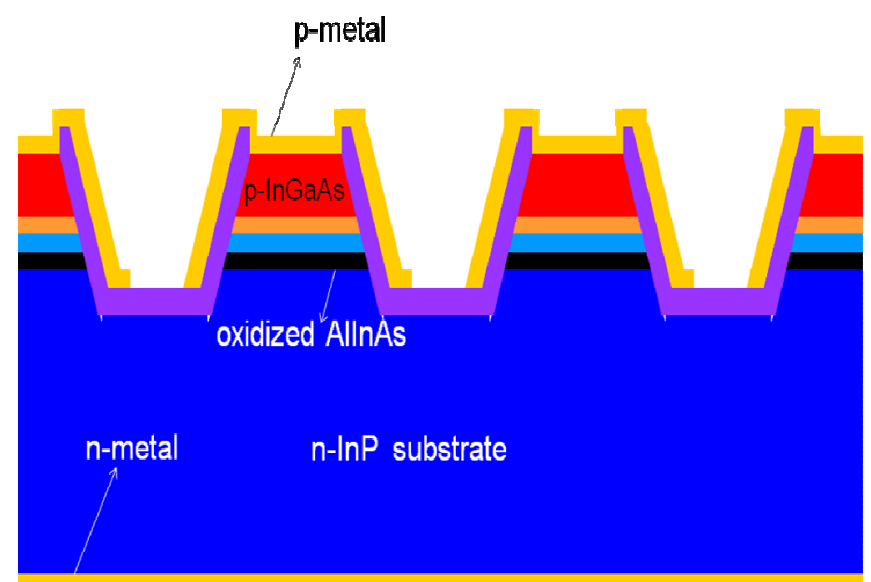

Fig.3. Schematic view of the device for electrical measurement

enough resistance is what we need to find out. Fig. 3 shows the schematic view of the $p$-i-n diode used to measure the resistance of the AllnAs oxide.

\subsection{I-V (Current-Voltage) measurement:}

Fig.4 shows the I-V curve for the unoxidized sample. The "turn-on" effect of the $p-n$ junction is clearly visible, it isaround 0.7 volts. Above turn on the resistance is around $25 \Omega$. Fig. 5 shows the I-V if the AllnAs layer $(100 \mathrm{~nm})$ is completely oxidized. The resistance of AllnAs oxide is at least $100 \mathrm{k} \Omega$, so that that wet oxidation brought an increase in the resistance of more than 3 orders.

4 Conclusion: The results indicate that AllnAs oxide in an InP membrane based photonic crystal laser is suitable for current blocking. This technique, combined with submicron selective area re-growth, is promising for an electrical injected $\operatorname{InP}$ membrane based photonic crystal lasers with low threshold currents and low power consumptions, as well as high output power.

\section{Acknowledgements}

The authors thank EU Project HISTORIC for financial support.

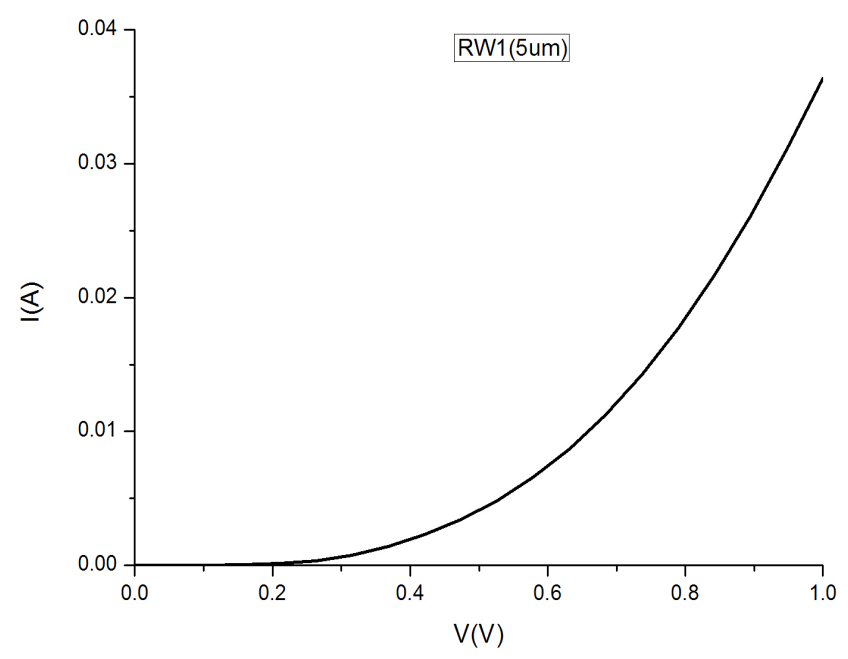

Fig. 4. I-V measurement of the unoxidized sample.

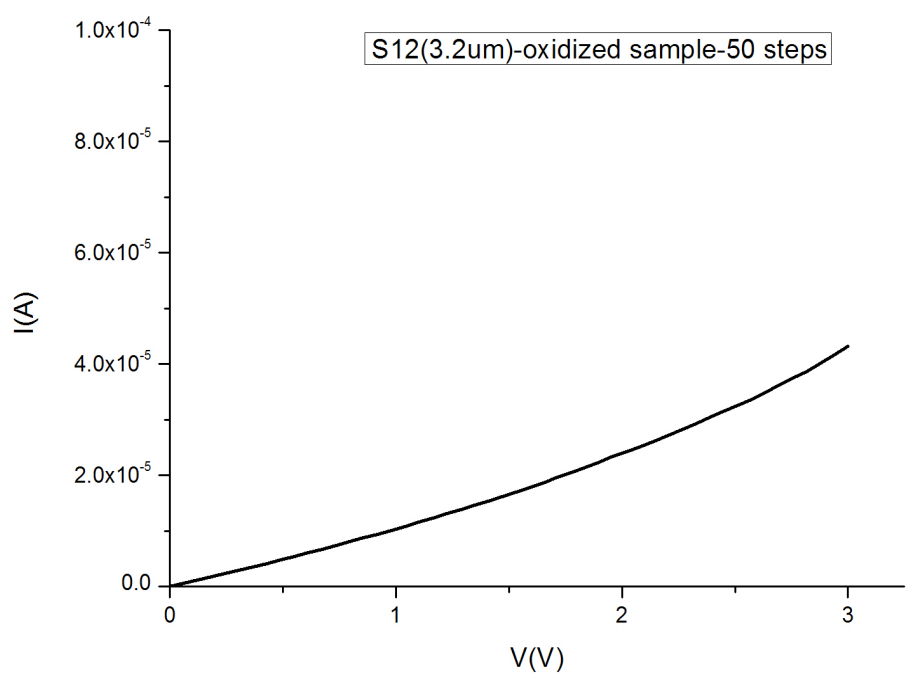

Fig. 5. I-V measurement of the oxidized sample.

\section{References}

[1] H. Gebretsadik, K. Kamath, W-D. Zhou, and P. Bhattacharya, "Lateral oxidation of InAlAs in InP-based heterostructures for long wavelength vertical cavity surface emitting laser applications," Appl. Phys. Lett. 72 (2), 12 January 1998

[2] D. L. Huffaker,O. Baklenov, L. A. Graham, B. G. Streetman, and D. G. Deppe ,"Quantum dot vertical-cavity surface-emitting laser with a dielectric aperture," Appl. Phys. Lett. 70 (18), 5 May 1997

[3] R. Zhang, F. Bordas, J.J.G.M. van der Tol, H. Ambrosius, G. Roelkens, M.A.Dundar and M.K. Smit, "Submicron Active-Passive Integration for InP-based Membranes on Silicon", Proc. ECIO 2010, Cambridge (UK), paper $\mathrm{ThH} 1$.

[4] J.J.G.M. van der Tol, R. Zhang, J.Pello, F. Bordas, G. Roelkens. H. Ambrosiusa, P. Thijs, F. Karouta and M. K. Smit, "Photonic Integration in Indium-Phosphide Membranes on Silicon," IET Optoelectronics, in press 